the problems that had to be faced in designing an automatic ticketing system in three areas in Belgium, namely, Brussels, Antwerp and Malines. The urban network of Brussels contains fifteen exchanges of the rotary type, some dating back to 1922. The normal capacity is 115,000 lines, and this network furnishes a good example of the manner in which automatic ticketing can be added to existing equipments. The author states that Antwerp, Liége, Charleroi and other Belgian towns will follow the method adopted for Brussels.

The first installation of automatic ticketing was put into service in Bruges in 1936, and was rapidly followed by installations in Ostend and twelve other towns in Belgium. During the period July 1-September 15, 1937, a check of 62,000 ticketed calls revealed 80 tickets, or $0 \cdot 13$ per cent, bearing incomplete or incorrect details, and these results go far to prove that full-automatic methods can be applied to long-distance service with the same success as to local service.
The tickets provide the traffic department with valuable information, and also give a remarkably clear picture of the operating efficiency of the exchange. Each long-distance call is recorded whether it be an effective connexion or whether it results in "busy", "no answer", "wrong number", or "premature release". Equipment faults which might otherwise remain unsuspected and undetected are brought to light by the indisputable evidence of the ticket. Naturally, further developments in this field are to be anticipated. It may prove advantageous to use a small number of high-speed printers which are engaged only at the termination of a call.

A form of ticket may be produced which can be automatically sorted, and, ultimately, doubtless mechanized-accounting will further dispense with the need for human intervention. Finally, it is anticipated that automatic ticketing will prove immensely valuable in Europe when the development of international long-distance service on a fully automatic basis becomes possible.

\title{
ANALYSIS OF FIELD TRIALS
}

$\mathrm{V}$ ARIETY testing forms an essential part of the work of the plant breeder and so he needs an adequate technique for the design and statistical analysis of field trials. His requirements in this direction are, however, rather specialized, the prime necessity being a method of comparing a large number of selected lines, though the careful testing of a small number of valuable new strains against standard varieties is also of importance.

Now one of the greatest advances made by modern statistics has been to revolutionize field trial technique. Until recently, however, the development of field designs was largely concerned with manurial and other such trials, where a limited number of treatments are applied and where interactions of these treatments are detectable and potentially of interest. Furthermore, when it was shown that multiple interactions are seldom if ever significant, a new technique, that of confounding, became possible. A design involving confounding purposely sacrifices information on less important interactions, in order to make the main comparisons with more precision.

Clearly all field trials must be based on the same principles as these 'treatment' designs; but the latter in themselves are not fully suited to the plant breeder's work. He often requires to work with large numbers of varieties and has no uninteresting 'interaction' comparisons which may be sacrificed by simple confounding. His requirements have, however, recently received special attention, and appropriate lay-outs have been developed. For example, the quasi-factorial designs, involving a somewhat complicated application of confounding, meet the needs of the plant breeder admirably but are in general of small value to those interested in soil treatment.

Thus it is very appropriate that the Imperial Bureau of Plant Breeding and Genetics should publish a technical communication on the subject of field trials, by an author who has been associated for many years with this branch of statistical work*. The publication is essentially introductory in scope, and the contents have been admirably chosen to fulfil this purpose. The first sections deal with the general principles of yield determination and the measurement of experimental error. Then follow descriptions of the randomized block and Latin square designs, together with details of their numerical analysis. The discussion of the use of the Latin square well illustrates the considerations involved in the choice of a design appropriate to an experiment. Multiple factor experiments are next dealt with and the principles of orthogonality and confounding introduced by a simple example. Finally, the split plot technique, which is especially suited to combined variety and manurial trials, and the quasi-factorial designs are briefly considered. The latter is somewhat complex for introduction into an elementary account, but its inclusion is amply justified by its pre-eminent position among layouts for testing a large number of varieties.

The presentation of the material is adequate, though more open to criticism than is the choice of contents, in that it tends at times to be too technical. For example, the introduction of the terms 'selfconjugate' and 'transformation set' into the section on Latin squares is unnecessary as their meanings are not explained, the reader being referred elsewhere for an account of their use. Thus they do not materially aid the argument and are somewhat terrifying to the beginner. The discussion of the $t$ test in the third section could also be made much more real for the biologist by the use of actual experimental data as illustrative matter, rather than the very artificial example of two varieties with unequal means but exactly equal variances, which is in fact used.

There can, however, be no doubt that plant breeders will find this publication a helpful introduction to the subject of field trials. Nor will its use end there, as the list of more advanced works given, together with short notes on their contents, in the last section, will serve as a guide for later reading and reference.

K. MATher. * Field Trials: Their Lay-out and Statistical Analysis. By John
Wishart. (Imperial Bureau of Plant Breeding and Genetics, Cambridge.) 